\title{
SmartGlove for Upper Extremities Rehabilitative Gaming Assessment
}

\author{
${ }^{\dagger}$ Ming-Chun Huang, `Wenyao Xu, $¥$ Yi Su, ` Belinda Lange, \\ "Chien-Yen Chang and ${ }^{\dagger}$ Majid Sarrafzadeh \\ + Wireless Health Institute, University of California, Los Angeles, \\ California, 90095, USA \\ ₹ Computer Science, Extension of University of California, Los Angeles, \\ California, 90095, USA \\ "Institute for Creative Technologies, University of Southern California, Los Angeles, \\ California, 90089, USA
}

\begin{abstract}
This paper presents a quantitative assessment solution for an upper extremities rehabilitative gaming application [1]. This assessment solution consists of a set of stand-alone hardware, including SmartGlove and Kinect, a depth capturing sensor made by Microsoft. SmartGlove is a specially designed motion and finger angle extraction device which is packaged in an easy-to-wear and adjustable manner for a patient with an upper extremity impairment. Sensor data extraction, alignment, and visualization algorithms were designed for integrating hand-mounted sensors data streams into skeleton coordinates captured by the Kinect. This enhanced skeleton information can be summarized and replayed as upper extremity joint coordinate animations which can be used for physical therapists to quantify rehabilitation progress. In addition, to serve as an assessment tool, enhanced skeleton information can be used to extend the capability of the Kinect vision system, such as providing motion capture of the upper extremities, even when the testing subject is out of camera scope or one's upper extremities are occluded by the body.
\end{abstract}

\section{Keywords}

Upper extremities, upper limbs, rehabilitation, video game, data glove, Kinect, animation.

\section{Categories and Subject Descriptors}

J.3 [Health and Medical information systems]: LIFE AND MEDICAL SCIENCE.

\section{General Terms}

Design, Economics, Reliability, Experimentation.

\section{INTRODUCTION}

Permission to make digital or hard copies of all or part of this work for personal or classroom use is granted without fee provided that copies are not made or distributed for profit or commercial advantage and that copies bear this notice and the full citation on the first page. To copy otherwise, to republish, to post on servers or to redistribute to lists, requires prior specific permission and/or a fee. PETRA'12, June 6 - 8, 2012, Crete Island, Greece. Copyright @ 2012 ACM ISBN 978-1-4503-1300-1/12/06... \$10.00
According to U.S heart disease and stroke statistics 2012 report, the direct and indirect health care cost of stroke is approximately $\$ 34.3$ billion in 2008 . Around 795,000 people suffer from a new stroke or recurrent stroke every year. On average, $\$ 7657$ is spent per person for stroke care in U.S [2]. This information reflects that strokes are one of the major health care expense sources and stroke can be considered a severe burden on both the U.S government and individual families affected by stroke. In addition to medicine-oriented fees, people with stroke often need physical therapy services to improve stroke symptoms or retain functionality and independence with daily activities. The loss of function, particularly in the upper extremities, can be recovered via effective limb rehabilitation [3]. However, the stroke rehabilitation process is usually time-consuming and tedious. With a lack of suitable and immediate motivation, some patients even quit their rehabilitation in a short period of time.

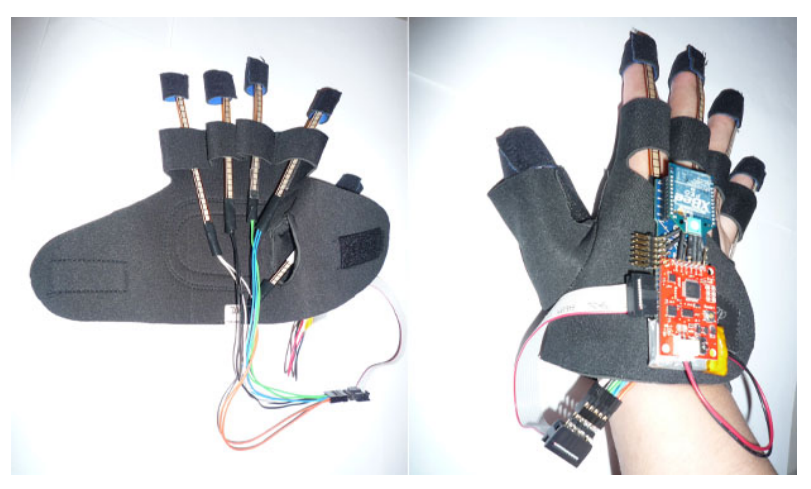

Figure 1: New SmartGlove Design

One potential reason for a rise in health care costs is because people often do not follow their rehabilitation programs through to the end. In addition, to correctly identify rehabilitation progress, there usually needs to be a professional physical therapist involved. However, the limited number of qualified physical therapists decreases the availability of the service and increases the treatment expenses. On the other hand, examining rehabilitation progress with only limited quantitative and subjective tools reduces the possibility of comparing the progress similarities and differences between individual people with stroke. Therefore, 
several researchers have started to introduce commercially available electronics with task-specific visual feedback into rehabilitative treatments. Flynn et al. reports an individual post-stroke rehabilitation case with a popular gaming platform, Sony PlayStation2 [3]. Lange et al. adopts Microsoft Kinect for upper limbs reaching tests with special designed virtual characters [4]. Their research transforms rehabilitation processes into interactive games with low-cost and commercial available hardware which facilitates the effort of deployment in the hospitals. Also, this setting is suitable for in-home deployment. With carefully designed data collection criterion, such as following Fugl-Meyer assessment requirements [5], and enabling remote data access, tele-rehabilitation could be a new option to effectively decrease therapy expenses and eliminate inconvenient travels to clinics for disabled patients.

\section{BACKGROUND}

Tele-rehabilitation research has concentrated on camera-based data collection and analysis for a long time, because video cameras really enable doctors to see their patients and can record every detail of the patients' movements with continuous video monitoring. However, one negative aspect of this method is that data volume is usually too large to be analyzed and too much unrelated information interferes with patient activity research, such as environmental background. The invention of Microsoft Kinect provides a straightforward manner to analyze patient activities by extracting human joints/skeleton information from video streams. This information is considerably valuable in analyzing stoke patient's motion and extremities activities. However, the official Kinect development tools did not provide enough resolution for fingers' tracking [6], which are important for the hand grasping task of the Fugl-Meyer Motor assessment [5]. There are several solutions proposed to enable fine-grained finger tracking with Kinect. Z. Ren et al proposes a robust hand contour detection method to detect and track both hand and finger motions [7], but the proposed method seems unsuitable for time-critical applications, such as interactive rehabilitation games. Another way to track hands efficiently is via using extra hardware, such as a color glove. Through tracking the glove color, hand positions and gestures can be identified with image processing tools and algorithms [8]. Except for hand and finger position tracking, gloves can serve more versatile functionality. For example, Vutinuntakasame et al. designed a wireless data glove which equips five bending sensors to detect finger curling angles and capture detailed finger activities for American sign language applications [9]. Similar to the motion capture controller$\mathrm{s}$ in Wii, inertial motion unit (IMU) can be used to track hand motion as well. These added external sensors take advantage of direct measurements of hand motions and finger curling angles to eliminate the necessary image processing and feature extraction time for hand parameters tracking, though extra hardware is necessary. Another advantage of the wireless data gloves is that they are not limited by Kinec$\mathrm{t} /$ camera view field and do not prevent normal human movements. Nevertheless, some issues still exist. Wireless signal transmission tends to be absorbed by the human body; therefore, although wireless gloves will not have occlusion issues occurred in a camera-based system, the transmitted data packets may be delayed or disappear during the transmission [10]. The consequence of the loss of the packets is that glove-glove and glove-camera synchronization will break occasionally after data loss or delay. In this paper, we describe detailed SmartGlove hardware design considerations and data re-synchronizing algorithms to integrate camera and glove's data streams together. Detailed finger curling and hand position information are integrated with Kinect skeleton coordinates using IMU-enhanced iterative closest point algorithm [11]. Personal dead reckoning (PDR) algorithm [12] is applied when the hands of the test subject are out of camera view field or occluded by the body. The remainder of the paper is constructed as follows: Section 3 introduces the SmartGlove package design, system and animation replay setup. Section 4 explains the algorithms in sensor data synchronization and integration. Section 5 discusses an empirical evaluation of our system and approach. Section 6 summarizes and suggests future research directions.

\section{SYSTEM ARCHITECTURE 3.1 SmartGlove Package Design}

SmartGlove consists of five finger bending sensors, an IMU, an Xbee RF wireless communication module, an Arduino Fio microcontroller [13] and two velcro gloves. Fig.1 shows the gloves' appearance and their integrated sensor modules. We specially designed the glove architecture with FLEXIFIT manual wheelchair gloves [14]. Our previous studies with a female stroke patient demonstrated that even though a regular glove design [1] works well for a normal user, it may not be suitable for a stroke patient who could not separate her fingers well when she wore the gloves. In that study, the fitting process, putting on a pair of gloves, took more than 10 minutes and needed at least one person to help her to get through. The same problem happened when she took off the gloves. In addition, this female patient had long fingernails, so that she could not stretch her fingers fully to the end of the glove. Due to the fact that a bending sensor's reading is sensitive to the contact region of the user's finger, the changes of length of her fingernails slightly affected the sensor readings. Therefore, repeated calibration for every week use is required. We also received feedback for adopting a more flexible glove structure which allows a user to adjust the tightness of the glove at the wrist region and adjust the location of the bending sensors for different finger length. Our new design attempts to satisfy all these requirements. With velcro straps attached around the wrist, the tightness of the gloves can be adjusted. When a SmartGlove is put on a table and the velcro straps are unwrapped, a user can easily put his/her hand into the glove and stretch his/her fingers step by step until all fingers slide into the correct finger positions. We designed a flexible fabric ring with velcro on each bending sensor to make the bending sensor position adjustable. This fabric ring design avoids the long fingernails issue and makes the new SmartGloves adjustable for the different length of the fingers. We utilize XBee RF transmitters and receivers to communicate data from the gloves with a laptop, but the communication media is not limited to RF only, the communication media can be replaced with Bluetooth or WiFi by changing the current transmitters and receivers with the corresponding communication modules. We tested different communication media, but in our experiment, we choose XBee because it can easily measure and configure its transmission power strength, which is required for our data synchronization algorithm design and 
it can avoid unwanted human interference, such as bluetooth connectivity problems and router configuration mistakes.

\subsection{Kinect Skeleton Descriptions}

Microsoft provides official software development kit (SDK), which supports human skeleton coordinates based on the captured 640 by 480 32-bit RGB images and the corresponding 320x240 16-bit depth map. Kinect SDK provides up to 20 joints tracking per person, but limited field of view and depth sensor ranges restrict the object tracking range of Kinect. These joints include the head, body, and extremities. There are several software packages existing which exploit sophisticated image processing techniques and join$\mathrm{t}$ analysis algorithms to extend the number of the tracking joints. Our goal is to add one subject finger bending information on top of the existing skeleton structure provided by Kinect SDK. Although Kinect can support multiple user tracking, our current implementation did not include this feature for simplicity.

\subsection{Animation Replay}

After having all the skeleton coordinates provided by Kinect API and computed bent finger coordinates, we designed a sequence of coordinates trajectories replay in Maya [15] and visualized the joints as a virtual character animation. Animation replay helps physical therapists to reexamine the rehabilitation process and check details in the targeted upper extremities movements. Instead of displaying IMU and bending sensor data in the waveform format, skeleton-based animation replay facilitates therapists' work to identify the problems of the patients in the rehabilitation process. Moreover, patients can easily understand their current progress and problems via watching their skeleton animation replay. Visualized information can assist both therapists and patients to figure out their progress intuitively. Fig. 2 shows three snapshots to describe how the animation replay works. In fact, the size of the joint skeleton information is far smaller than the corresponding video streams. Therefore, animation replay is considerably time and memory efficient even with a remote skeleton information accessing.

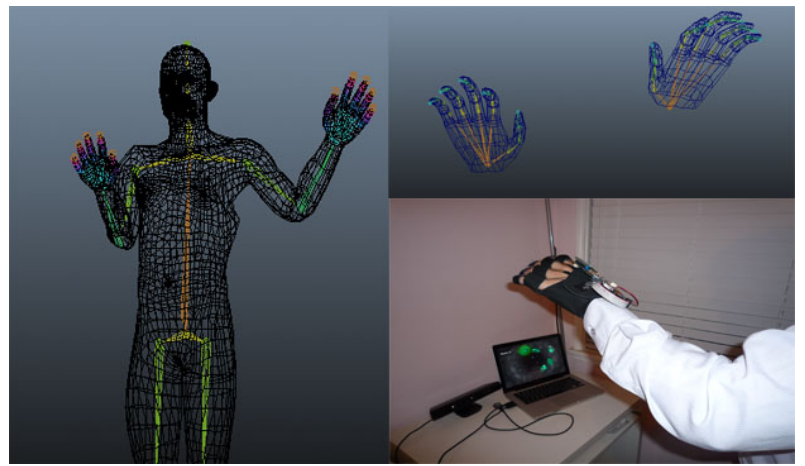

Figure 2: Animation

\section{ALGORITHM FOR USER IDENTIFICA- TION}

Algorithm framework is shown in Fig. 3. First of all, a laptop connected with Kinect via Kinect specific power supply cable collects all Kinect skeleton data for each frame. An
XBee receiver connected to the laptop collects IMU data and all five bending sensors data. IMU data consists of $x$, $y, z$ axis acceleration, pitch, roll, and yaw orientation data. Low pass filtering are applied to the IMU sensor data to remove noise. Initial calibration is performed to compute IMU static offsets and build an angle conversion table for each finger. Although bending sensor datasheet provides their calibration circuit and conversion formula, we still computed the table empirically in the initial calibration step to make sure that the transduce finger angles are reliable and accurate. The same game application, virtual gem grasping game (JewelMine), $[1,4]$ is adopted in this paper. We observed that every subject tends to reach and then statically grasps the targeted gems before he/she moves to the virtual basket in the game. Therefore, at each statically grasping moment, where the bending sensor values are close to 90 degree and the Euclidean distance of the 3-axis accelerometer readings approximately equals to the gravity, we adopt Bas des Bouvrie [11] proposed method to align IMU coordinates with Kinect coordinates.

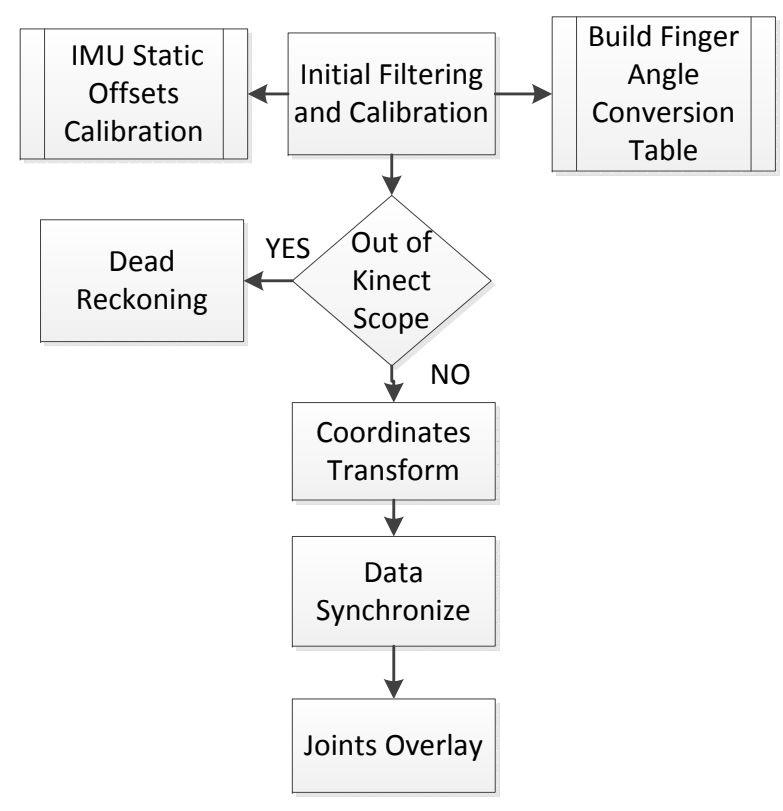

Figure 3: Algorithm Flowchart

If the IMU orientation data is far from the hand skeleton orientation of Kinect after alignment. XBee Received Signal Strength Indication (RSSI) is measured and the transmission module is required to increase its transmission power to decrease data loss which causes IMU orientation data to be inconsistent with Kinect hands joint orientation. If both SmartGlove and Kinect orientations match within our empirically designed threshold, we treat the whole upper extremities tracking system as synchronized. If not, the current Kinect frames, IMU, and bending sensor data are marked as unsynchronized as offline records. If the whole system is synchronized, we add finger coordinates onto Kinect hand coordinates and the full skeleton coordinates are stored and served for offline animation replay.

\section{EVALUATION}


A preliminary study recruited three subjects to go through this game-guided rehabilitation. One subject is a male who had no disability in his upper extremities. The other two subjects, one male and one female, have slight upper extremity impairment, but still can move their hands and reach the virtual gems in the game. This evaluation setup was designed to test if the finger angle information can help in reaching and grasping rehabilitation. Due to the limitation of Kinect view field, we required the test subject$\mathrm{s}$ to stand or sit 1 meter away from Kinect. Each subject was asked to finish two rounds of the reaching and grasping game. Each round consisted of eight gems. The gem locations and the grasping orders are adjustable and followed the suggestion of physical therapists. While the subjects follow the visual hints in the game, their hand parameters, skeleton, and time stamp information are recorded. After the session was completed, the stored information was replayed to visualize the rehabilitation process. Three observations of the experiments were made. First of all, PDR algorithm only worked for a short period of time. The tracking coordinates started to deviate apparently after five seconds out of camera range. Nevertheless, the Glove orientation data were still in the consistent orientation even though the SmartGloves is not in the Kinect view field. This is because the IMU module purchased from Sparkfun can correct its sensor reading deviation with attitude and heading reference system (AHRS) coding constantly. Its accelerometer and digital compass modules utilize gravity and earth's magnetic field as references to calculate pitch, roll, and yaw angles to correct sensor values. Last, we found out that the current glove design can capture finger movements, however, it still has difficulties in measuring the space between a pair of adjacent fingers. However, this feature is not required in this gem reaching and grasping gaming application.

\section{CONCLUSION AND FUTURE WORK}

The proposed upper extremities rehabilitation assessment system provides quantified and visualized information to evaluate upper extremities rehabilitation progress. Detailed finger bending angle information is integrated seamlessly with Kinect captured joint information. A large volume of video streams are summarized by $\sim 30$ joint coordinates.

The next step of this research will focus on large scale clinical trials to obtain more general and pervasive feedback. Furthermore, to reduce repeated work in device calibration, on-line automatic calibration should be implemented in the gaming design, such as a creating finger angle table dynamically in the game. Integrating game scenes with calibration steps should effectively reduce the demand of the initial calibration and postpone the calibration step until the game starts.

\section{ACKNOWLEDGEMENTS}

This work is partially supported by NSF Grant IIS- 1238660 .

\section{REFERENCES}

[1] M. Huang and E. Chen and W. Xu and M. Sarrafzadeh, "Gaming for upper extremities rehabilitation," in Wireless Health, p. 27, ACM Press, 2011.
[2] American Heart Association, "Heart disease and stroke statistics-2012 update," Circulation, vol. 125, no. 1, pp. e12-e230, 2012.

[3] S. Flynn and P. Palma and A. Bender, "Feasibility of using the sony playstation 2 gaming platform for an individual poststroke: A case report," Journal of neurologic physical therapy, vol. 31, no. 4, pp. 180-189, 2007.

[4] B. Lange and E. Suma and B. Newman and T. Phan and C. Chang and A. Rizzo and M. Bolas, "Leveraging Unencumbered Full Body Control of Animated Virtual Characters for Game-Based Rehabilitation," in Proceedings of Human Computer Interface, pp. 243-252, HCI Press, 2011.

[5] A. Fugl-Meyer and L. Jaasko and I. Leyman and S. Olsson and S. Steglind, "The post-stroke hemiplegic patient a method for evaluation of physical performance," Scandinavian journal of rehabilitation medicine, vol. 7, no. 1, pp. 71-78, 1975.

[6] C. R. Cameron and L. W. Divalentin and R. Manaktala and A. C. McElhaney and C. H. Nostrand and O. J. Quinlan and L. N. Sharpe and A. C. Slagle and C D. Wod and Y. Z. Yang and G. J Gerling, "Hand tracking and visualization in a virtual reality simulation," in Systems and Information Engineering Design Symposium, pp. 127-132, IEEE Press, 2011.

[7] Z. Ren and J. Yuan and Z. Zhang, "Robust Hand Gesture Recognition Based on Finger-Earth Mover Distance with a Commodity Depth Camera," in Proceedings of the 19th ACM international conference on Multimedia, pp. 1093-1096, ACM Press, 2011.

[8] I. Oikonomidis and N. Kyriazis and A. Argyros, "Efficient Model-based 3D Tracking of Hand Articulations using Kinect," in Proceedings of the British Machine Vision Conference, pp. 101.1-101.11, BMVA Press, 2011.

[9] S. Vutinuntakasame and V. R. Jaijongrak and S. Thiemjarus, "An Assistive Body Sensor Network Glove for Speech- and Hearing-Impaired Disabilities," in Body Sensor Networks, 2011 International Conference on, pp. 7-12, IEEE Press, 2011.

[10] N. Amini and W. Xu, Z. Li, M. Huang, M. Sarrafzadeh, "Experimental Analysis of IEEE 802.15.4 for On/Off Body Communications," in IEEE 22nd International Symposium on Personal Indoor and Mobile Radio Communications, pp. 2138-2142, IEEE Press, 2011.

[11] B. d. Bouvrie, "Improving RGBD Indoor Mapping with IMU data," Master's thesis, Delft University of Technology, 2011.

[12] L. Ojeda and J. Borenstein, "Personal Dead-reckoning System for GPS-denied Environments," in IEEE International Workshop on Safety, Security, and Rescue Robotics, pp. 1-6, IEEE Press, 2007.

[13] "News - SparkFun Electronics." http://www. sparkfun. com/.

[14] "Grover Gear Medical - home of Wheelchair Tire Covers-Wheel Chair Gloves." http://www. grovergear. com/servlet/StoreFront.

[15] "Maya - 3D animation software - Autodesk." http://usa.autodesk. com/maya/. 